\title{
Introduction to the Minitrack on Health Technologies that Interface Patients and Providers
}

\author{
Kai Zheng \\ University of California, Irvine \\ kai.zheng@uci.edu
}

\author{
Yunan Chen \\ University of California, Irvine \\ yunanc@uci.edu
}

\begin{abstract}
This HICSS minitrack is devoted to a class of health technologies that serve as an interface between patients and their medical providers. These technologies not only help patients gain easier access to their own medical records, but also help providers obtain patient-generated data as well as extend their care services to patient homes. If designed and used properly, these technologies have the potential to facilitate patient-provide communication, and bridge the knowledge, information, and data gap between patients and medical professionals. Based on the peerreview feedback, we selected four papers to include in this minitrack. These papers cover a wide range of topics, from evaluating the effectiveness of specific applications to observational and focus group studies aiming to obtain a better understanding of sociotechnical issues and privacy concerns surrounding the use of such applications. We look forward to further research development on this important topic.
\end{abstract}

\section{Introduction}

With electronic health records now being widely available across U.S. hospitals and physician offices, there is an impetrative need to extend the capability and reach of clinically-oriented information systems to better engage patients in their own care. Such extensions can provide patients with more effective tools to manage their health. They also allow patients to better communicate with their providers, view and act upon their medical data, and generate rich health information in their everyday activities that can be shared with their providers for more informed medical decision making.

This minitrack solicited submissions that focus on information and technological innovations bringing patients and providers together to fulfill each other's unmet needs. Examples of such innovations include patient portals that provide patients downloadable data in addition to actionable insights; computer-mediated communication technologies designed to facilitate patient-provider interactions; as well as applications that support patient-generated data, which can be shared with medical professionals and used seamlessly in their clinical workflow. In this minitrack, we invited submissions of all types of research, including and not limited to theoretical development, conceptual design, prototyping, laboratory experiments, implementation science studies, and evaluations and clinical trials. The scope of the research could be:

- Studies of extensions of clinical IT systems that can be directly used by patients, caregivers, or the general public;

- Studies of computer-mediated communication technologies that engender more rapid and more effective provider-provider interactions;

- Studies of applications that enable patients to generate health data in their everyday living and share the data with their healthcare providers to inform clinical reasoning and medical decision making;

- Studies of technologies that enable novel patient care delivery methods, such as eVisits, telehealth through affordable technologies, and home-based care that allows medical professionals to monitor patient conditions and intervene from remote;

- Studies of online innovations that allow patients to perform self-service activities such as finding out waiting time of ED or walk-in clinics; selfdiagnosis and self-triage assisted by medical professionals; and personal health decision making.

\section{Accepted Papers}

Out of many excellent submissions received, we selected four papers to eventually include in the minitrack.

In the first paper, entitled "Can Hospital's Analytics Capabilities Impact Patient Satisfaction? A Multi-Year Panel Study" [1], Adjerid, Angst, \& Devaraj used the organization information processing theory to examine the effect of healthcare analytics on hospital performance. The empirical study was based on 
multiple sources of panel data, and focused on two types of healthcare analytics applications: business intelligence and data mining. Their study used patient satisfaction as the main outcome measure. The results show that having healthcare analytics capabilities is positively associated with patient satisfaction, which is moderated by clinical complexity and concentration. This paper establishes an important first step toward answering an interesting, yet underexplored question regarding the benefits of having healthcare analytics capabilities in provider organizations.

In another paper, "The Impact of Computing Device Design on Patient-Centered Communication: An Experimental Study" [2], Randell et al. report a study assessing the impact of different computing devices on patient-provider communication. More specifically, they conducted an empirical study to compare desktop, tablet, and tabletop devices using a within-participant experimental study. Their findings suggest that the choice between these devices has a strong influence on the levels of patient-centeredness, in addition to the openness of patient-provider conversations and the privacy concerns raised. Based on these findings, the authors suggested that a large, adjustable horizontal screen is more desired to facilitate eye contact and patient engagement.

In the third paper, entitled "Effect of Handoff Training on Resident Communication Quality: An Observational Study" [3], Abraham et al. reports an observational study evaluating the impact of a handoff training program on communication quality. The outcomes were measured in the form of frequency of breakdowns. The authors conducted a qualitative analysis of the content of audio-recorded handoff conversations, followed by a statistical analysis that compared the behavior of residents assigned to the control versus the training group. The findings suggest that there are inherent differences in the clinical content communicated between the study groups. Residents assigned to the training group discussed more intensively on identifying information, contextual information, and past medical history; whereas residents assigned to the control group covered more on active problems and assessment.

In the fourth paper, "Investigating Ethical Design Requirements for Digitalized Healthcare Support: The Case of Ambulatory Physiotherapeutic Assistance

Systems," Mueller, Heger, and Niehaves conducted a focus group study that involved developers, scientists, and physicians to understand ethical and social challenges in the context of developing an ambulatory physiotherapeutic assistance system. The authors performed a qualitative analysis of the focus group results, and reported their findings under four main themes: privacy considerations, user autonomy, user competence, and designs for accommodating the heterogeneity of all users. This study points to the importance of considering patient autonomy, privacy, competence, and social-cultural issues in designing health interface technologies.

\section{References}

[1] Idris Adjerid, Corey Angst, Sarv Devaraj. Can hospital's analytics capabilities impact patient satisfaction? A multiyear panel study. The 51st Hawaii International Conference on System Sciences (HICSS-51). Big Island, Hawaii, 2018.

[2] Rebecca Randell, Charlotte Tang, Yunan Chen. The impact of computing device design on patient-centered communication: An experimental study. The 51st Hawaii International Conference on System Sciences (HICSS-51). Big Island, Hawaii, 2018.

[3] Joanna Abraham, Thomas Kannampallil, Charlotte Ward, Christopher Bogan, Abbas Hyderi. Effect of handoff training on resident communication quality: An observational study. The 51st Hawaii International Conference on System Sciences (HICSS-51). Big Island, Hawaii, 2018.

[4] Marius Mueller, Oliver Heger, Bjoern Niehaves. Investigating ethical design requirements for digitalized healthcare support: The case of ambulatory physiotherapeutic assistance systems. The 51st Hawaii International Conference on System Sciences (HICSS-51). Big Island, Hawaii, 2018. 